Palavras chave: Colorimetria Qualidade da madeira Sistema CIE L*a*b*.

Histórico:

Recebido 17/08/201 I

Aceito 16/12/2013

Keywords:

Colorimetry

Wood quality

CIE L*a*b* system

Correspondência: rosileigar@ufrrj.br

DOI:
Rosilei Aparecida Garcia' , Nathalia Silveira de Oliveira', Alexandre Miguel do Nascimento', Natália Dias de Souza'

\section{COLORIMETRIA DE MADEIRAS DOS GÊNEROS Eucalyptus E Corymbia E SUA CORRELAÇÃO COM A DENSIDADE}

RESUMO: Conduziu-se, este estudo, com os objetivos de caracterizar a cor da madeira de Corymbia citriodora (Hook.) K.D. Hill \& L.A.S. Johnson, Eucalyptus pellita F. Muell, Eucalyptus paniculata Sm. e Eucalyptus grandis Hill ex Maiden; e determinar sua correlação com a densidade da madeira, visando a avaliar o potencial da colorimetria como uma ferramenta para a identificação e qualificação da madeira. As análises colorimétricas foram realizadas segundo o sistema CIE L*a*b* com o auxílio do espectrofotômetro portátil CM 2600d da Konica Minolta. Os resultados indicaram diferenças significativas entre as densidades das espécies estudadas, sendo a madeira de Eucalyptus paniculata a mais densa e a madeira de Eucalyptus grandis a menos densa. As análises de correlação entre a cor e a densidade monstraram que as madeiras mais densas, para as espécies do gênero Eucalyptus estudadas, são mais escuras (menor $L^{*}$ ) e apresentam mais pigmento vermelho (a*) e amarelo (b*). Entretanto, o comportamento foi diferente para o Corymbia citriodora, cuja madeira apresentou alta densidade, cor clara e pouco pigmento vermelho, indicando que a combinação das variáveis colorimétricas e da densidade apresenta potencial para segregar a espécie Corymbia citriodora das demais espécies de Eucalyptus estudadas. As madeiras mais densas de Eucalyptus pellita são mais escuras na seção radial, enquanto que as madeiras de Eucalyptus paniculata apresentaram mais pigmento amarelo na seção tangencial. Cada espécie apresentou uma coloração específica, dada pelas variáveis colorimétricas, mostrando o potencial da colorimetria na identificação de madeiras

\section{COLORIMETRY OF WOODS FROM Eucalyptus AND Corymbia GENUS AND ITS CORRELATION WITH DENSITY}

ABSTRACT: The objectives of this study were to characterize the wood color of Corymbia citriodora (Hook.) K.D. Hill \& L.A.S. Johnson, Eucalyptus pellita F. Muell, Eucalyptus paniculata Sm. and Eucalyptus grandis Hill ex Maiden; and to determine its correlationship with wood density in order to evaluate the potential of colorimetry as a tool for identification and qualification of wood. Color analysis were performed on the CIE L*a*b* system by using the CM 2600d spectrophotometer from Konica Minolta. The results indicated significant differences between wood densities of all species. Eucalyptus paniculata wood presented the highest density while Eucalyptus grandis wood presented the lowest density. Correlation analysis between color and density showed that the denser woods, considering the species from the Eucalyptus genus studied, are darker (lower $\left.\mathrm{L}^{*}\right)$ and present redder ( $\left.\mathrm{a}^{*}\right)$ and more yellow ( $\left.\mathrm{b}^{*}\right)$ hues. However, the behavior was different for Corymbia citriodora, whose wood had high density, light color and little red hue, indicating that color variables and density combination has potential to segregate the Corymbia citriodora species from other Eucalyptus species studied. The denser woods of Eucalyptus pellita are darker in the radial section, while woods of Eucalyptus paniculata presented more yellow hue $\left(b^{*}\right)$ in the tangential section. Each species presented a specific color, given by colorimetric variables, showing the potential of colorimetry for wood identification. 


\section{INTRODUÇÃO}

A utilização de madeiras de reflorestamento, principalmente do gênero Eucalyptus, vem crescendo, em razão do melhor conhecimento de suas propriedades, da maior divulgação, da crescente preocupação com a preservação das florestas nativas e do valor comercial competitivo, comparado às madeiras nativas (FERREIRA et al., 2004; VALENÇA et al., 2002).

O gênero Eucalyptus, descrito por L'Héritier, pertence à família Myrtaceae e conta com cerca de seiscentas espécies e grande número de variedades e híbridos, sendo a maioria das espécies originária da Austrália (ANDRADE, I96I). As madeiras de Eucalyptus sp. apresentam uma grande variedade de cores, sendo o alburno claro e o cerne, variando desde um amarelado a bege (Eucalyptus dunni, Eucalyptus maculata, Eucalyptus nitens, Eucalyptus globulus) até tons de pardoavermelhados à vermelhos (Eucalyptus robusta, Eucalyptus pellita, Eucalyptus grandis, Eucalyptus saligna, Eucalyptus tereticornis, Eucalyptus resinifera), e densidades aparentes variando de 0,40 à I,20 g.cm (MUÑIZ, 2002).

Estudos baseados na análise filogenética e nas características morfológicas e moleculares do gênero Eucalyptus têm causado discussões e controvérsias sobre a reclassificação desse gênero. Hill e Johnson (1995) incluíram algumas espécies de Eucalyptus no gênero Corymbia, sendo inseridas 113 espécies, entre elas o Eucalyptus citriodora Hook. ( = Corymbia citriodora (Hook.) K.D. Hill \& L.A.S. Johnson). Neves (2008) observou, por meio de estudos de Ressonância Magnética Nuclear de Carbono 13 (RMN de ${ }^{13} \mathrm{C}$ ), que a madeira de Corymbia citriodora apresentou sinais característicos de deslocamento químico dos carbonos, diferentes daqueles encontrados para o gênero Eucalyptus, confirmando a necessidade da reclassificação para o gênero. Por outro lado, na classificação proposta por Brooker (2000), o Eucalyptus citriodora está inserido no gênero Eucalyptus, subgênero Corymbia. Ladriges e Udovicic (2000) concordam com a classificação proposta por Hill e Johnson (1995) e discordam de Brooker (2000). Segundo Ladiges e Udovicic (2000), a classificação de Brooker (2000) é contrária às evidências filogenéticas baseadas em dados morfológicos, análises do polimorfismo no comprimento de fragmentos de restrição (RFLPs - Restriction Fragment Length Polymorphisms) e sequências de dados de DNA. Portanto, no presente trabalho, optou-se por utilizar a nomenclatura Corymbia citriodora (Hook.) K.D. Hill \& L.A.S. Johnson.
De acordo com Ribeiro (2009), a colorimetria pode ser utilizada para a identificação e qualificação da madeira. Apesar da cor ser uma característica tão importante quanto às propriedades físicas e mecânicas da madeira, ela não tem sido tão discutida quanto às demais características, tais como: propriedades físicas, mecânicas, químicas e anatômicas. Segundo Nishino et al. (2000), conhecimentos sobre a relação da cor com outras características físicas da madeira, pode contribuir para a compreensão do comportamento da cor, além de determinar uma utilização mais adequada para determinadas espécies.

Quando associada a outras características organolépticas, tais como textura e desenho, a cor da madeira facilita a identificação da espécie e indica a sua melhor forma de utilização. Segundo Möttönen et al. (2002), as características da madeira e a homogeneidade da cor têm desempenhado um importante papel para estabelecer os preços da madeira serrada. Por essa razão, essa propriedade deve ser incorporada à caracterização tecnológica da madeira, para atender aos usos mais nobres desse material (MORI et al., 2005).

A cor da madeira pode ser influenciada por fatores genéticos e ambientais, sendo este o mais importante em termos de impacto na variação da cor (RINK, 1987). A cor natural da madeira depende de vários fatores, tais como: espécie, densidade, teor de umidade, teor de extrativos, estrutura anatômica, taxa de crescimento, idade da árvore e tratamentos silviculturais (GIERLINGER et al., 2004; KLUMPERS et al., 1993; MOSEDALE et al., 1996; RINK; PHELPS, 1989; SOTELO MONTES et al., 2008).

A densidade da madeira é uma das propriedades físicas mais importantes, sendo utilizada como indicador de qualidade. Burger e Richter (199I) afirmam que, geralmente, as madeiras mais escuras são mais densas e mais resistentes que as madeiras mais claras.

Objetivou-se, neste trabalho, caracterizar a cor da madeira de Corymbia citriodora (Hook.) K.D. Hill \& L.A.S. Johnson, Eucalyptus pellita F. Muell, Eucalyptus paniculata Sm e Eucalyptus grandis Hill ex Maiden; e determinar sua correlação com a densidade da madeira visando a avaliar o potencial da colorimetria como uma ferramenta para a identificação e qualificação da madeira.

\section{MATERIAL E MÉTODOS}

\section{Material}

As espécies utilizadas neste estudo foram Corymbia citriodora (Hook.) K.D. Hill \& L.A.S. Johnson 
(I7 anos de idade), Eucalyptus pellita F. Muell. (17 anos de idade), Eucalyptus paniculata Sm. (65 anos de idade) e Eucalyptus grandis Hill ex Maiden (23 anos de idade). Foram utilizadas quatro árvores, sendo uma de cada espécie, as quais foram coletadas em um plantio localizado no campus da Universidade Federal Rural do Rio de Janeiro (UFRRJ) em Seropédica, RJ. Dessas árvores, retiraram-se amostras aleatórias do cerne, a partir da altura do DAP, sendo 6 de cada espécie, totalizando 24 amostras com dimensões de $30 \times 30 \times$ $50 \mathrm{~mm}$ perfeitamente orientadas nos planos transversal, longitudinal radial e longitudinal tangencial.

\section{Determinação da densidade aparente}

Adensidade aparente da madeira foi determinada de acordo com a norma D 2395 da American Society for Testing and Materials - ASTM (1999).

\section{Determinação da cor no sistema CIE L*a*b*}

A cor da madeira foi determinada segundo o sistema CIE L*a*b*, o qual trabalha de acordo com a norma da Commission Internationale de L'Eclairage - CIE (1976) e é composto por três coordenadas: L* representando a luminosidade ou claridade no eixo preto-branco, a qual varia de zero (preto) a 100 (branco), $a^{*}$ e b* representando as coordenadas cromáticas nos eixos verde-vermelho e azul-amarelo, respectivamente, ambas variando entre -60 e +60 .

As variáveis $L^{*}, a^{*}$ e b* foram obtidas com o auxílio do espectrofotômetro portátil CM 2600d (Konica Minolta) disponível no Departamento de Produtos Florestais (Instituto de Florestas, UFRRJ). As medições foram efetuadas por meio do contato direto da superfície da amostra com a área de visão do aparelho, com diâmetro de abertura de $3 \mathrm{~mm}$ (SAV - small area view), utilizando-se o iluminante padrão D65 e ângulo de observação de $10^{\circ} \mathrm{com}$ luz especular incluída. Antes das medições, as superfícies das amostras foram polidas com lixas de granulometria de 120 e 220.

Para cada amostra, foram efetuadas 10 medições, em pontos equidistantes, sendo 5 medições na seção radial (L*radial, a*radial e b*radial) e 5 na seção tangencial ( $L$ *tang., a*tang. e b*tang.), para cada variável. Os dados coletados pelo espectrofotômetro foram transferidos para o software SpectraMagic ${ }^{\mathrm{TM}} \mathrm{NX}$ (CM-SI00w), versão I.9, da Konica Minolta e, então, exportados para o software Microsoft Office EXCEL para as análises estatísticas.
Os valores de saturação da cor (C) e ângulo de tinta (h*) foram calculados para a caracterização da cor das espécies pelas seguintes equações:

$C=\sqrt{(a *)^{2}+(b *)^{2}}$

$h^{*}=\tan ^{-1}\left(\frac{b^{*}}{a^{*}}\right)$

\section{Análises estatísticas}

Os dados de densidade foram submetidos a uma análise de variância (ANOVA) com o auxílio do programa $S A S^{\circledR}$ System, a fim de determinar as diferenças significativas. Quando o valor $\mathrm{F}$ foi significativo $(\alpha=0,05)$, aplicou-se $o$ teste de média de Duncan $(\alpha=0,05)$.

As variáveis $L^{*}, a^{*}$ e $b^{*}$ nas seções radial e tangencial das quatro espécies, não apresentaram distribuição normal dos dados nem homogeneidade das variâncias. A transformação das variáveis foi aplicada, entretanto, não foi possível obter a normalidade dos dados. A partir de então, a análise estatística foi feita pelo método de Kruscal-Wallis e as diferenças estatísticas entre as médias dos postos foram feitas pelo teste de Bonferroni $(\alpha=0,05)$.

\section{RESULTADOS E DISCUSSÃO}

\section{Densidade da madeira}

Os resultados obtidos para a densidade aparente das madeiras de Corymbia citriodora, Eucalyptus pellita, Eucalyptus paniculata e Eucalyptus grandis são apresentados na Tabela I.

As análises estatísticas indicaram diferenças significativas entre as densidades da madeira das quatro espécies. A madeira de Eucalyptus paniculata apresentou a maior densidade $\left(1,060{\mathrm{~g} . \mathrm{cm}^{-3}}^{-3}\right.$, seguida das madeiras de Corymbia citriodora $\left(0,840 \mathrm{~g} \cdot \mathrm{cm}^{-3}\right)$, Eucalyptus pellita $(0,804$ $\left.\mathrm{g} . \mathrm{cm}^{-3}\right)$ e Eucalyptus grandis $\left(0,508 \mathrm{~g} . \mathrm{cm}^{-3}\right)$. De acordo com a literatura, as densidades aparentes (a 15\% de umidade) das madeiras de Corymbia citriodora e Eucalyptus grandis são de 1,040 e $0,500 \mathrm{~g} . \mathrm{cm}^{-3}$, respectivamente, enquanto que para a madeira de Eucalyptus pellita, a densidade aparente (a I2\% de umidade) é de 0,995 g.cm ${ }^{-3}$ (DOMBRO, 2010; INSTITUTO DE PESQUISAS TECNOLÓGICAS DO ESTADO DE SÃO PAULO - IPT, 2003). Para a madeira de Eucalyptus paniculata, a densidade básica é de I, $120 \mathrm{~g} \cdot \mathrm{cm}^{-3}$ (NEVES et al., 2004). 
TABELA I Valores médios obtidos para a densidade aparente e as variáveis de cor no sistema CIE L*a*b*, nas seções radial e tangencial, das madeiras de Corymbia citriodora, Eucalyptus pellita, Eucalyptus paniculata e Eucalyptus grandis.

TABLE I Means values obtained for density and color variables on CIE L*a*b* system, on radial and tangential sections, of Corymbia citriodora, Eucalyptus pellita, Eucalyptus paniculata and Eucalyptus grandis woods.

\begin{tabular}{|c|c|c|c|c|c|c|c|c|c|c|c|c|}
\hline \multirow{2}{*}{ Espécie } & \multirow{2}{*}{$\begin{array}{c}\text { Densi } \\
\text { dade } \\
\left(\mathrm{g} \cdot \mathrm{cm}^{-3}\right)\end{array}$} & \multicolumn{2}{|c|}{ L* } & \multicolumn{2}{|c|}{$a^{*}$} & \multicolumn{2}{|c|}{$b^{*}$} & \multicolumn{2}{|c|}{$C$} & \multicolumn{2}{|c|}{$\mathrm{h}^{*}$} & \multirow{2}{*}{$\begin{array}{c}\text { Cor da } \\
\text { madeira' }\end{array}$} \\
\hline & & Radial & Tang. & Radial & Tang. & Radial & Tang. & Radial & Tang. & Radial & Tang. & \\
\hline $\begin{array}{l}\text { Corymbia } \\
\text { citriodora }\end{array}$ & $\begin{array}{l}0,840^{\mathrm{b}} \\
{[2,38]}\end{array}$ & $\begin{array}{l}67,6^{\mathrm{bA}} \\
(147)\end{array}$ & $\begin{array}{l}65,8^{\mathrm{bA}} \\
(120)\end{array}$ & $\begin{array}{c}6, I^{d A} \\
(33,5)\end{array}$ & $\begin{array}{c}6,0^{C A} \\
(27,6)\end{array}$ & $\begin{array}{l}19,9^{\mathrm{bA}} \\
(131,8)\end{array}$ & $\begin{array}{l}19, I^{\mathrm{bA}} \\
(102)\end{array}$ & $\begin{array}{l}20,9^{\mathrm{bA}} \\
(30,5)\end{array}$ & $\begin{array}{l}20,0^{\text {cA }} \\
(27,3)\end{array}$ & $\begin{array}{c}73,0^{\mathrm{aA}} \\
(105,5)\end{array}$ & $\begin{array}{c}72,7^{\mathrm{AA}} \\
(103,5)\end{array}$ & $\begin{array}{l}\text { Cinza- } \\
\text { rosada }\end{array}$ \\
\hline $\begin{array}{c}\text { Eucalyptus } \\
\text { pellita }\end{array}$ & $\begin{array}{l}0,804^{c} \\
{[2,48]}\end{array}$ & $\begin{array}{l}68,2^{\mathrm{bA}} \\
(156,7)\end{array}$ & $\begin{array}{l}63,6^{\text {bВ }} \\
(93,5)\end{array}$ & $\begin{array}{c}15,7^{\mathrm{bA}} \\
(153,2)\end{array}$ & $\begin{array}{c}16,4^{\mathrm{aA}} \\
(174,3)\end{array}$ & $\begin{array}{c}22,3^{\mathrm{aA}} \\
(215,9)\end{array}$ & $\begin{array}{c}21,2^{\mathrm{aA}} \\
(178,9)\end{array}$ & $\begin{array}{l}27,2^{\mathrm{aA}} \\
(97,6)\end{array}$ & $\begin{array}{c}26,8^{\mathrm{aB}} \\
(102,7)\end{array}$ & $\begin{array}{l}54,9 c A \\
(45,5)\end{array}$ & $\begin{array}{l}52,3^{\mathrm{cB}} \\
(43,3)\end{array}$ & Rosada \\
\hline $\begin{array}{l}\text { Eucalyptus } \\
\text { paniculata }\end{array}$ & $\begin{array}{l}I, 060^{\mathrm{a}} \\
{[0,94]}\end{array}$ & $\begin{array}{l}54,5^{\mathrm{cA}} \\
(43,4)\end{array}$ & $\begin{array}{l}5 I, 2^{\mathrm{CA}} \\
(2 I, 2)\end{array}$ & $\begin{array}{c}17,6^{\mathrm{aA}} \\
(216,2)\end{array}$ & $\begin{array}{l}16,5^{\mathrm{aA}} \\
(178,3)\end{array}$ & $\begin{array}{l}19,8^{\mathrm{bA}} \\
(130,4)\end{array}$ & $\begin{array}{l}18,0^{\text {bв }} \\
(60,1)\end{array}$ & $\begin{array}{l}26,5^{\mathrm{A}} \\
(83,4)\end{array}$ & $\begin{array}{l}24,5^{\mathrm{bB}} \\
(73,2)\end{array}$ & $\begin{array}{l}48,3^{\mathrm{dA}} \\
(15,5)\end{array}$ & $\begin{array}{l}47,6^{\mathrm{dB}} \\
(15,7)\end{array}$ & $\begin{array}{c}\text { Marrom- } \\
\text { avermelhada }\end{array}$ \\
\hline $\begin{array}{c}\text { Eucalyptus } \\
\text { grandis }\end{array}$ & $\begin{array}{l}0,508^{d} \\
{[1,97]}\end{array}$ & $\begin{array}{l}72,4^{\mathrm{aA}} \\
(203)\end{array}$ & $\begin{array}{l}69,7^{\mathrm{aA}} \\
(179)\end{array}$ & $\begin{array}{l}10,8^{\mathrm{cA}} \\
(88,4)\end{array}$ & $\begin{array}{l}11,0^{\mathrm{bA}} \\
(92,5)\end{array}$ & $\begin{array}{l}18,0^{c A} \\
(60,0)\end{array}$ & $\begin{array}{l}18,6^{\mathrm{bA}} \\
(84,8)\end{array}$ & $\begin{array}{l}21,0^{\mathrm{bA}} \\
(30,5)\end{array}$ & $\begin{array}{l}21,6^{\mathrm{cA}} \\
(36,7)\end{array}$ & $\begin{array}{l}59, I^{\mathrm{bA}} \\
(75,5)\end{array}$ & $\begin{array}{l}59,6^{\mathrm{bA}} \\
(73,5)\end{array}$ & $\begin{array}{c}\text { Rosa- } \\
\text { acinzentada }\end{array}$ \\
\hline Valor $F$ & $\begin{array}{c}538,48 \\
p<0,01\end{array}$ & - & - & - & - & - & - & - & & - & & \\
\hline
\end{tabular}

Os valores entre colchetes referem-se aos coeficientes de variação e os valores entre parênteses às médias dos postos feitos pelo teste de Kruscal-Wallis. As letras minúsculas comparam as médias dentro da mesma coluna e as letras maiúsculas entre colunas, para a mesma variável, sendo que as médias com letras iguais não são estatisticamente diferentes entre si pelo teste de Bonferroni $(\alpha=0,05)$. A comparação das médias de densidade aparente foi feita pelo teste de Duncan $(\alpha=0,05)$. 'Classificação de cor proposta por Camargos (I999).

Apesar dos valores de densidade encontrados neste estudo serem similares àqueles encontrados na literatura, é importante mencionar que a idade das espécies estudadas pode ter influência nos valores de densidade. A maior densidade obtida para a madeira de Eucalyptus paniculata, principalmente quando comparada ao Corymbia citriodora, pode ter sido afetada pela presença de madeira adulta, já que essa espécie apresentava uma idade de, aproximadamente, 65 anos, enquanto que as demais espécies apresentavam idades entre 17 e 23 anos, com predominância de madeira juvenil. Palma et al. (2010) estudaram a madeira de Corymbia citriodora, com 29 anos de idade, e observaram que a madeira juvenil aparece até o a metade do raio, sendo a outra metade, mais externa, pertencente a madeira madura.

\section{Caracterização colorimétrica da madeira}

Os valores médios de L* (luminosidade) mostram que, para ambas as seções radial e tangencial, a madeira de Eucalyptus grandis é a mais clara (maior L*), enquanto que a madeira de Eucalyptus paniculata é a mais escura (menor L*). As espécies de Eucalyptus pellita e Corymbia citriodora apresentaram valores de L* estatisticamente iguais com valores de 68,2 e 67,6 na seção radial e 63,6 e 65,8 na seção tangencial, respectivamente. Comparando os valores de $L^{*}$ entre as seções radial e tangencial, observam-se menores valores na seção tangencial para todas as espécies estudadas, entretanto, somente a espécie de Eucalyptus pellita apresentou diferenças significativas (Tabela I).
A coordenada $a^{*}$ (vermelho), na seção radial, apresentou valores estatisticamente diferentes para as quatro espécies estudadas, enquanto que, na seção tangencial, a madeiras de Eucalyptus pellita e Eucalyptus paniculata apresentaram valores similares (Tabela I). A madeira de Eucalyptus paniculata foi a espécie que apresentou mais pigmento vermelho (maior $\mathrm{a}^{*}$ ), enquanto que a madeira de Corymbia citriodora foi a espécie que apresentou menos pigmento vermelho (menor $\mathrm{a}^{*}$ ).

A coordenada b* (amarelo) apresentou menores variações entre os valores médios das espécies estudadas que a coordenada $\mathrm{a}^{*}$ (vermelho). Pode-se inferir que a coordenada a* é a principal responsável pela alteração de cor entre as espécies e faces de cada espécie, apesar da participação não menos importante da coordenada b* para compor a cor final da espécie. Na seção radial, a madeira de Eucalyptus pellita apresentou mais pigmento amarelo ( $b^{*}$ radial $=22,3$ ) quando comparada às demais espécies, enquanto que a madeira de Eucalyptus grandis apresentou menos pigmento amarelo ( $b *$ radial $=18,0$ ). Provavelmente, a cor rosada da madeira de Eucalyptus pellita é dada, em decorrência da participação equilibrada entre as duas coordenadas. Por outro lado, a cor rosaacinzentada da madeira de Eucalyptus grandis pode ser atribuída, principalmente, pela participação da coordenada $b^{*}$ e valores menores de $a^{*}$, em relação à espécie anterior. As espécies de Corymbia citriodora e Eucalyptus paniculata apresentaram valores de $b *$ radial similares. $\mathrm{Na}$ seção tangencial, somente a madeira de Eucalyptus pellita apresentou um valor de $b$ *tang. significativamente diferente 
das demais, sendo também a madeira com mais pigmento amarelo (maior $b^{*}$ tang.). Esses resultados indicam que as maiores variações de $a^{*}$ e $b^{*}$ entre as espécies ocorreram na seção radial. Comparando os valores de b*, entre as seções radial e tangencial, apenas a madeira de Eucalyptus paniculata apresentou diferença significativa, tendo a seção radial mais pigmento amarelo. A cor marrom-avermelhada dessa espécie é dada, principalmente, em função dos valores elevados de $\mathrm{a}^{*}$ (Tabela I).
Segundo a classificação proposta por Camargos (1999), as cores das madeiras de Corymbia citriodora, Eucalyptus pellita, Eucalyptus paniculata e Eucalyptus grandis podem ser descritas como cinza-rosada, rosada, marromavermelhada e rosa-acinzentada, respectivamente, considerando-se os valores encontrados para as variáveis $L^{*}$, a*, b*, C e h*. Na Figura I, apresenta-se a combinação das variáveis de cor $L^{*}, a^{*}$ e $b^{*}$ nas seções radial e tangencial para as quatro espécies. Pode-se observar que cada espécie
(A) Corymbia citriodora

(B) Eucalyptus pellita

(C) Eucalyptus paniculata

(D) Eucalyptus grandis
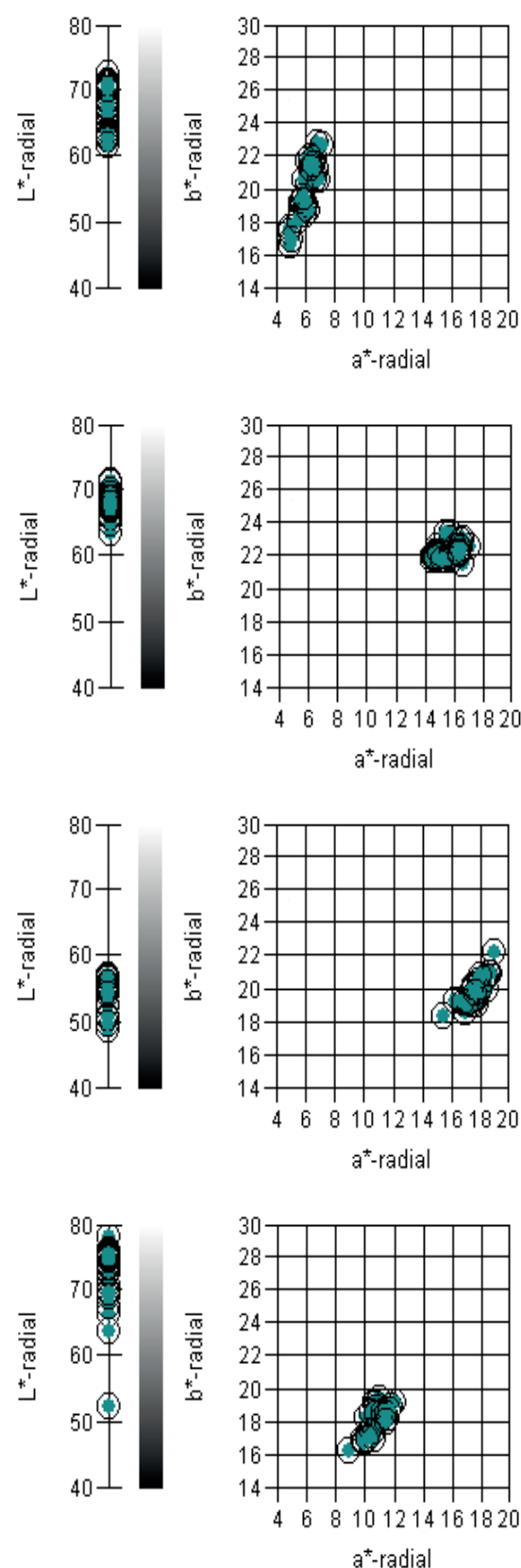
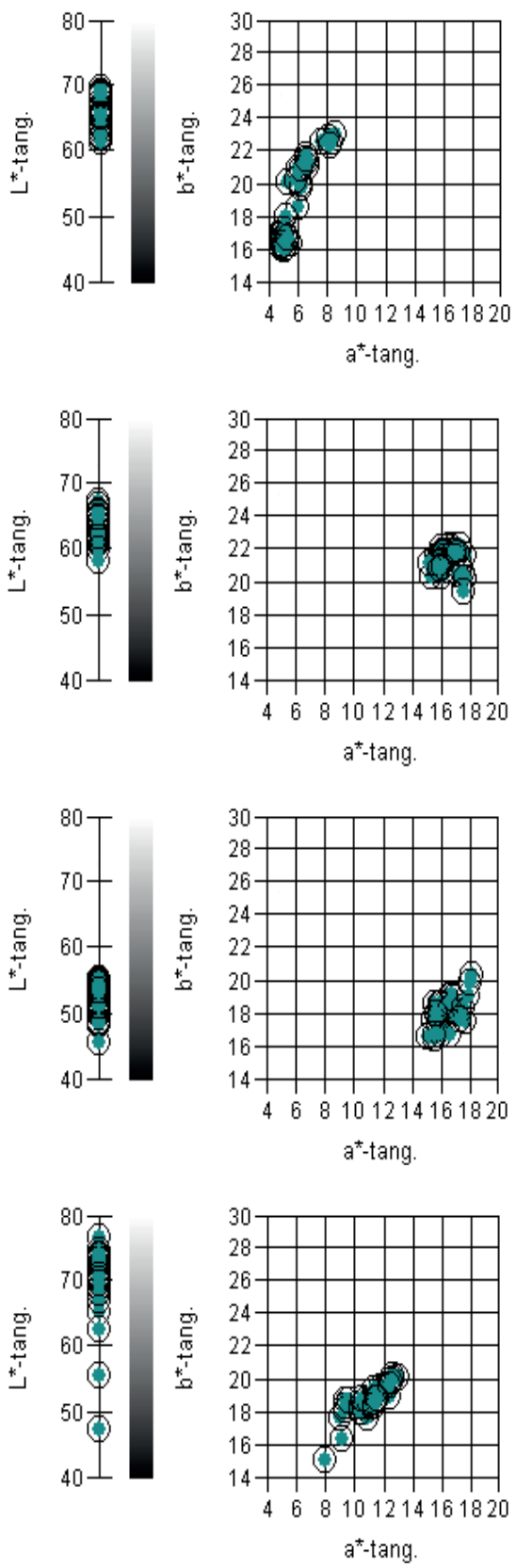

FIGURA I Variáveis de cor das madeiras de Corymbia citriodora, Eucalyptus pellita, Eucalyptus paniculata e Eucalyptus grandis nas seções radial e tangencial, respectivamente.

FIGURE I Color variables of Corymbia citriodora, Eucalyptus pellita, Eucalyptus paniculata and Eucalyptus grandis woods on radial and tangential sections, respectively. 
apresentou um padrão de cor diferente, indicando que a colorimetria tem potencial para ser utilizada como uma ferramenta de suporte para a identificação das espécies.

Entretanto, essas comparações devem ser realizadas com precaução, sendo necessárias mais pesquisas, já que a cor pode variar de acordo com as condições climáticas do local de crescimento, densidade, teor de umidade, teor de extrativos, taxa de crescimento, idade da árvore, posição de amostragem na árvore e tratamentos silviculturais (GIERLINGER et al., 2004; KLUMPERS et al., 1993; MOSEDALE et al., 1996; RINK; PHELPS, 1989; SOTELO MONTES et al., 2008).

Apesar do grande número de fatores afetando a cor, um estudo realizado por Vanclay et al. (2008) sobre a variação da cor da madeira de Eucalyptus dunnii Maiden, mostrou pouca variação entre árvores e dentro da mesma árvore, mostrando que os fatores externos não descaracterizam a cor da espécie.

\section{Correlações entre a densidade e as variáveis de cor da madeira}

$\mathrm{Na}$ Tabela 2, apresentam-se as análises de correlação de Pearson entre a densidade aparente da madeira e as variáveis de cor ( $L^{*}, a^{*}$ e $\left.b^{*}\right)$ nas seções longitudinal radial e longitudinal tangencial, para todas as espécies. As análises de correlação de Pearson entre a densidade e as variáveis de cor mostraram significâncias entre: (a) Dens $\times$ L*radial: correlação negativa significativa ( $r=-0,8857)$, isto é, a densidade aumentou à medida que o valor de L*radial diminuiu; (b) Dens $\mathrm{x}$ $L$ *tang.: correlação negativa significativa $(r=-0,8606)$. Em razão da correlação (densidade $x$ luminosidade) ser negativa para ambas as faces, isso indica que as madeiras mais densas são também as mais escuras; (c) Dens $x$ b*radial: uma correlação positiva significativa ( $r=0,4074)$ foi observada, indicando que as madeiras mais densas apresentam mais pigmento amarelo nessa face; e (d) Dens x a*radial: uma correlação positiva significativa ( $r=0,4479)$ foi observada, indicando que as madeiras mais densas apresentam mais pigmentos vermelhos nessa face.

Das correlações significativas observadas na Tabela 2, foram feitos gráficos (Figura 2A-F) onde pode-se observar que as correlações existentes não são lineares e nem linearizáveis. A partir disso, foram estudados modelos estatísticos para melhor representar o fenômeno da relação entre a densidade e as variáveis de cor e observar o grau de associação, por meio dos coeficientes de determinação $\left(R^{2}\right)$.
TABELA 2 Análises de correlação de Pearson entre a densidade e as variáveis de cor no sistema CIE L*a*b* para as quatro espécies.

TABLE 2 Pearson's correlation between density and color variables on CIE L*a*b* system for four wood species.

\begin{tabular}{|c|c|c|c|c|c|}
\hline \multirow{4}{*}{$\begin{array}{l}\text { Longitudinal } \\
\text { Radial }\end{array}$} & & Densidade & L*radial & a*radial & b*radial \\
\hline & L*radial & $-0,8857^{\star \star *}$ & I & - & - \\
\hline & a*radial & $0,4479^{*}$ & $-0,5924^{* *}$ & I & - \\
\hline & b*radial & $0,4074^{*}$ & $-0,1105$ & 0,3589 & I \\
\hline \multirow{4}{*}{$\begin{array}{l}\text { Longitudinal } \\
\text { Tangencial }\end{array}$} & & Densidade & L*tang. & a*tang. & b*tang. \\
\hline & $\mathrm{L}$ tang. & $-0,8606^{* *}$ & I & - & - \\
\hline & a*tang. & 0,3527 & $-0,5845^{\star *}$ & I & - \\
\hline & b*tang. & $-0,1025$ & 0,2252 & 0,2649 & I \\
\hline
\end{tabular}

** Significativo à $99 \%$ de probabilidade.

* Significativo à $95 \%$ de probabilidade

Na Figura 2D, a qual apresenta a dispersão dos dados de densidade em função de a*radial, observou-se que o modelo de regressão polinomial de segundo grau forneceu um melhor coeficiente de determinação $\left(R^{2}=\right.$ 0,9246). A não-linearidade dos dados ocorreu, em razão da inclusão da espécie Corymbia citriodora na análise, o qual apresentou alta densidade $\left(0,840 \mathrm{~g} \cdot \mathrm{cm}^{-3}\right)$ e pouco pigmento vermelho representado pela coordenada $a^{*}$, destacando-se das demais espécies do gênero Eucalyptus, nas quais a densidade aumentou, à medida que $\mathrm{a}^{*}$ (cor vermelha) aumentou. Esses resultados indicam que a combinação dessas variáveis é capaz de segregar a espécie Corymbia citriodora das demais espécies de Eucalyptus estudadas.

Para as análises de correlação linear entre as variáveis de cor, foram observadas significâncias entre $L^{*}$ e a* para ambas as seções radial e tangencial (Tabela 2). Entretanto, após a análise do gráfico de dispersão (Figura 2E-F), verificou-se que os modelos de regressão polinomial quadrática forneceram os melhores coeficientes de determinação com $R^{2}=0,8437$ e $R^{2}=$ 0,5480 para as seções radial e tangencial, respectivamente. Esses resultados indicam que as madeiras mais densas, para as espécies do gênero Eucalyptus estudadas, são mais escuras (menor $L^{*}$ ) e apresentam mais pigmento vermelho ( $\left.a^{*}\right)$ e amarelo ( $\left.b^{*}\right)$. Entretanto, isso não se aplica a espécie de Corymbia citriodora, cuja madeira é densa, de cor clara e com menos pigmento vermelho.

As análises de correlação também mostraram que, na seção radial, os coeficientes de determinação entre a densidade e as variáveis de cor são melhores que na seção tangencial, quando se comparam todas as espécies. Ribeiro (2009) também observou que, na 
A

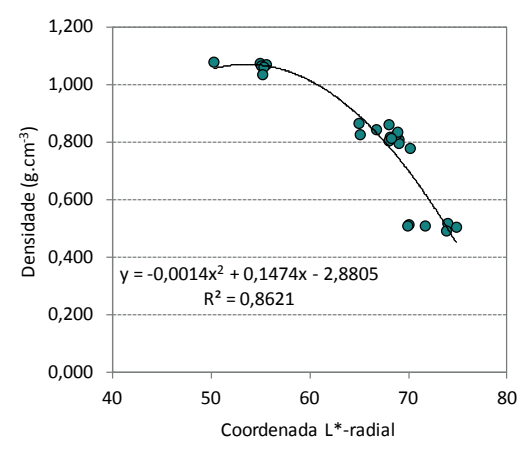

D

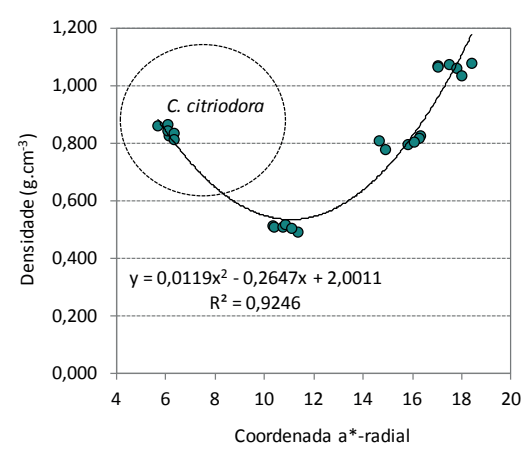

B

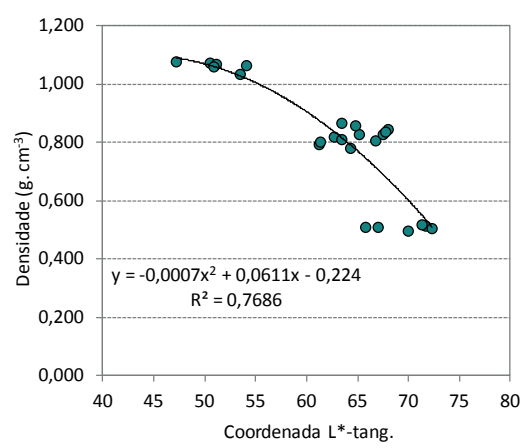

$\mathrm{E}$

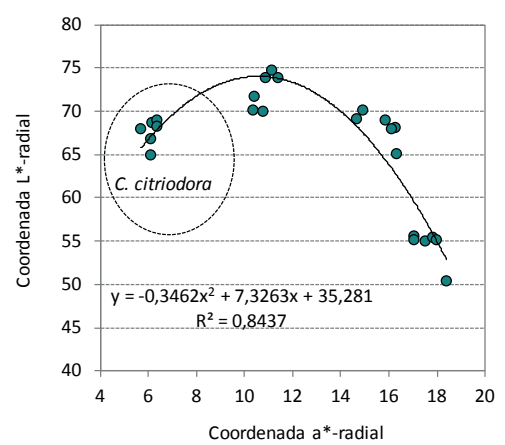

C

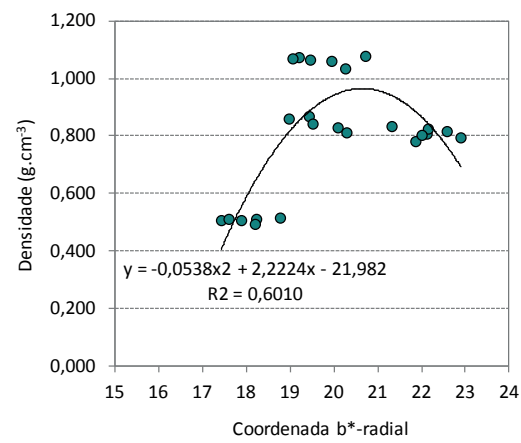

$\mathrm{F}$

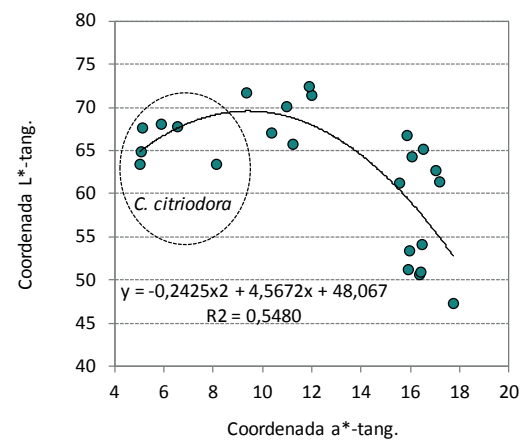

FIGURA 2 Dispersão dos dados de densidade em função das variáveis de cor (L*radial, L*tang., b*radial e a*radial) e da variável L* (luminosidade) em função da variável a* (vermelho) nas seções radial e tangencial, respectivamente, para as quatro espécies estudadas.

FIGURE 2 Data dispersion of the density as a function of the color variables (L*radial, L*tang., b*radial and a*radial) and L* (lightness) variable as a function of the $\mathrm{a}^{*}$ (red) variable on radial and tangential sections, respectively, for four species studied.

face radial, as correlações entre cor e densidade para a madeira de Eucalyptus grandis foram melhores.

$\mathrm{Na}$ Tabela 3, apresentam-se as análises de correlação de Pearson entre a densidade aparente da madeira e as variáveis de cor ( $L^{*}, a^{*}$ e b*) nas seções longitudinal radial e longitudinal tangencial, para cada espécie. A densidade apresentou uma correlação linear significativa com as variáveis de cor somente para as espécies de Eucalyptus pellita e Eucalyptus paniculata. Para a espécie Eucalyptus pellita, a densidade da madeira apresentou uma correlação negativa significativa com $L *$ radial $(r=-0,8629)$, indicando que as madeiras mais densas dessa espécie são mais escuras (menor $L^{*}$ ).

Para a espécie Eucalyptus paniculata, a densidade da madeira apresentou uma correlação positiva significativa com a variável $b *$ tang. ( $r=0,8 \mid 58)$, indicando que as madeiras mais densas dessa espécie apresentam mais pigmento amarelo (b*) na seção tangencial. Estudos realizados por Vanclay et al. (2008) também mostraram correlações significativas entre a densidade e a coordenada b* da madeira de Eucalyptus dunnii Maiden, ou seja, a coordenada b* (coloração amarela) foi inversamente proporcional a densidade da madeira, indicando que as madeiras mais densas dessa espécie apresentavam menos amarelo. As diferentes correlações obtidas entre a densidade e b* podem ser explicadas pela cor característica de cada espécie. Assim, os resultados obtidos para a espécie Eucalyptus paniculata caracterizam uma madeira marrom-avermelhada (Tabela I), enquanto que a madeira de Eucalyptus dunnii Maiden apresenta uma cor bege (LOPES, 2007).

As análises de correlação entre a densidade e as variáveis de cor indicam que a colorimetria pode ser utilizada como uma ferramenta no controle de qualidade da madeira, já que a densidade é um indicador importante da resistência, da dureza e da durabilidade da madeira. Entretanto, cada espécie de madeira terá correlações específicas com as variáveis $L^{*}, a^{*}$ e b*, em decorrência da cor característica de cada uma.

As correlações de Pearson entre as variáveis de cor mostraram significâncias somente para as madeiras de Eucalyptus paniculata e Corymbia citriodora. Para a madeira de Eucalyptus paniculata, foram observadas correlações positivas significativas entre $a^{*}$ radial $x$ 
TABELA 3 Análises de correlação de Pearson entre a densidade e as variáveis de cor no sistema CIE L*a*b* para cada espécie.

TABLE 3 Pearson's correlation between density and color variables on CIE L*a*b* system for each species.

\begin{tabular}{|c|c|c|c|c|c|c|c|c|}
\hline \multirow[t]{2}{*}{ Espécies } & \multicolumn{4}{|c|}{ Longitudinal Radial } & \multicolumn{4}{|c|}{ Longitudinal Tangencial } \\
\hline & & L*radial & a*radial & b*radial & & L*tang. & $\mathrm{a}$ *tang. & b*tang. \\
\hline \multirow{3}{*}{ Corymbia citriodora } & Densidade & $-0,6905$ & $-0,7212$ & $-0,6252$ & Densidade & $-0,1639$ & $-0,777 \mid$ & $-0,7752$ \\
\hline & L*radial & 1 & 0,2517 & 0,5820 & L*tang. & I & $-0,1956$ & 0,1513 \\
\hline & a*radial & - & I & $0,8339^{*}$ & a*tang. & - & I & $0,9331^{* *}$ \\
\hline \multirow{3}{*}{ Eucalyptus pellita } & Densidade & $-0,8629^{*}$ & 0,5901 & 0,1587 & Densidade & 0,2410 & 0,4454 & 0,5762 \\
\hline & L*radial & I & $-0,7 \mid 22$ & $-0,0030$ & L*tang. & I & $-0,2705$ & 0,6846 \\
\hline & a*radial & - & I & 0,3921 & $\mathrm{a}$ tang. & - & I & $-0,1676$ \\
\hline \multirow{3}{*}{ Eucalyptus paniculata } & Densidade & $-0,4657$ & $-0,1069$ & $-0,2215$ & Densidade & $-0,6765$ & 0,5689 & $0,8158^{*}$ \\
\hline & L*radial & I & $-0,7 \mid 77$ & $-0,7 \mid 42$ & L*tang. & I & $-0,7537$ & $-0,5772$ \\
\hline & a*radial & - & I & $0,9248^{* *}$ & a*tang. & - & I & $0,8563^{*}$ \\
\hline \multirow{3}{*}{ Eucalyptus grandis } & Densidade & $-0,3 \mid 20$ & $-0,6628$ & 0,2251 & Densidade & 0,0715 & $-0,0043$ & $-0,0026$ \\
\hline & L*radial & I & $0,750 \mathrm{I}$ & 0,3954 & L*tang. & I & 0,1044 & $0,568 \mathrm{I}$ \\
\hline & a*radial & - & I & 0,2822 & $\mathrm{a}$ tang. & - & I & 0,7982 \\
\hline
\end{tabular}

** Significativo à $99 \%$ de probabilidade. * Significativo à $95 \%$ de probabilidade.

b*radial $(r=0,9248)$ e $\mathrm{a}^{*}$ tang. $\mathrm{x}$ b*tang. $(r=0,8563)$, indicando que as madeiras com mais pigmento vermelho ( $\left.\mathrm{a}^{*}\right)$ também apresentam mais pigmento amarelo (b*). O mesmo comportamento foi observado para a madeira de Corymbia citriodora, ou seja, observou-se uma correlação positiva entre as coordenadas cromáticas $a^{*}$ e b* para ambas as seções radial $(r=0,8339)$ e tangencial $(r=0,933 \mathrm{I})$ (Tabela 3).

\section{CONCLUSÕES}

As espécies estudadas apresentaram densidades da madeira significativamente diferentes, sendo a madeira de Eucalyptus paniculata a mais densa, seguida das madeiras de Corymbia citriodora, Eucalyptus pellita e Eucalyptus grandis.

Cada espécie apresentou uma coloração específica, dada pelas variáveis colorimétricas, indicando que a colorimetria pode ser utilizada como uma ferramenta de suporte para a identificação de madeiras.

As análises de correlação indicaram que as madeiras mais densas, para as espécies do gênero Eucalyptus, são mais escuras (menor $L^{*}$ ) e apresentam mais coloração avermelhada ( $\left.a^{*}\right)$ e amarelada ( $\left.b^{*}\right)$. Entretanto, isso não se aplica à espécie de Corymbia citriodora, cuja madeira é densa, de cor clara e com pouca coloração avermelhada.

A combinação das variáveis colorimétricas e da densidade apresenta potencial para segregar a espécie Corymbia citriodora das demais espécies de Eucalyptus estudadas.
As análises de correlação, para cada espécie, mostraram que as madeiras mais densas de Eucalyptus pellita são mais escuras (menor $L^{*}$ ) na seção radial, enquanto que as madeiras de Eucalyptus paniculata apresentaram mais pigmento amarelo ( $\left.b^{*}\right)$ na seção tangencial.

Sugere-se que nos estudos de colorimetria sejam incluídas as curvas de reflectância.

\section{REFERÊNCIAS}

AMERICAN SOCIETY FOR TESTING AND MATERIALS. D 2395: standard test methods for specific gravity of wood and wood-based materials. Philadelphia, 1999. 357 p.

ANDRADE, E. N. O eucalipto. 2. ed. Jundiaí: Cia Paulista de Estradas de Ferro, 1961. 667 p.

BROOKER, M. I. H. A new classification of the genus Eucalyptus L'Hér. (Myrtaceae). Australian Systematic Botany, Colingwood, v. I3, n. I, p. 79-148, 2000.

BURGER, M. B.; RITCHER, H. G. Anatomia da madeira. São Paulo: Nobel, 199I. 154 p.

CAMARGOS, J. A. A. Colorimetria quantitativa aplicada na elaboração de um catálogo de cores de madeiras tropicais. 1999. 75 f. Dissertação (Mestrado em Ciências Florestais) - Universidade de Brasília, Brasília, 1999.

COMMISSION INTERNATIONALE DE L'ÉCLAIRAGE. Colorimetry. $2^{\text {nd }}$ ed. Vienna, 1976.

DOMBRO, D. B. Eucalyptus pellita: Amazonia reforestation's red mahogany. Bogotá: Planeta Verde Reforestación, 2010. $8 \mathrm{p}$. 
FERREIRA, S.; LIMA, J. T.; ROSADO, S. C. S.; TRUGILHO, P. F. Influência de métodos de desdobro tangenciais no rendimento e na qualidade da madeira de clones de Eucalyptus spp. Cerne, Lavras, v. I0, n. I, p. I0-2 I, 2004.

GIERLINGER, N.; JACQUES, D.; GRADNER, M.; WIMMER, R.; SCHWANNINGER, M.; ROZENBERG, P.; PÂQUES, L. E. Colour of larch heartwood and relationships to extractives and brown-rot decay resistance. Trees, Vancouver, v. 18, p. 102-108, 2004.

HILL, K. D.; JOHNSON, L. A. S. Sistematic studies in the eucalyptus 7: a revision of the bloodwoods, genus Corymbia (Myrtaceae). Telopea, Sydney, v. 6, p. 173-505, 1995.

INSTITUTO DE PESQUISAS TECNOLÓGICAS DO ESTADO DE SÃO PAULO. Madeira: uso sustentável na construção civil. São Paulo, 2003. 59 p.

KUMPLERS, J.; JANIN, G.; BECKER, M. The influences of age, extractive content and soil water on wood color in oak: the possible genetic determination of wood color. Annales des Sciences Forestières, Champenoux, v. 50, n. I, p. 403-409, 1993.

LADIGES, P. Y.; UDOVICIC, F. Comment on a new classification of the Eucalypts. Australian Systematic Botany, Colingwood, v. I3, n. I, p. I49-I52, 2000.

LOPES, C. S. D. Caracterização da madeira de três espécies de eucalipto para uso em movelaria. 2007. 89 f. Dissertação (Mestrado em Recursos Florestais) Universidade de São Paulo, Piracicaba, 2007.

MORI, C. L. S. O. de; LIMA, J. T.; MORI, F. A.; TRUGILHO, P. F; GONCALEZ, J. C. Caracterização da cor da madeira de clones de híbridos de Eucalyptus spp. Cerne, Lavras, v. I I, n. 2, p. I37-I46, 2005.

MOSEDALE, J. R.; CHARRIER, B.; JANIN, G. Genetic control of wood colour, density and heartwood ellagitannin concentration in European oak (Quercus petraea and $Q$. robur). Forestry, Oxford, v. 69, n. 2, p. III-I24, 1996.

MÖTTÖNEN, K.; ALVILA, L.; PAKKANEN, T. CIELab measurements to determine the role of felling season, log storage and kiln drying on coloration of silver Birch wood. Scandinavian Journal Forest Research, Kalmar, v. I7, p. |79-19|, 2002.
MUÑIZ, G. I. B. Fatores que limitam a utilização da madeira de Eucalyptus. In: SEMINÁRIO SÓLIDOS DE EUCALIPTO: AVANÇOS CIENTÍFICOS E TECNOLÓGICOS, 2002, Lavras. Anais... Lavras: UFLA, 2002. p. 30-63.

NEVES, K. M. Estudo comparativo de classificação da madeira de Corymbia citriodora por RMN de ${ }^{13} \mathrm{C}$. 2008. 55 p. Monografia (Graduação em Engenharia Florestal) - Universidade Federal Rural do Rio de Janeiro, Seropédica, 2008.

NEVES, W. A. A.; COSTA, L. G.; BRAGA, E. P.; VIEIRA, I. G. Chave de identificação de espécies florestais (CIEF). Piracicaba: IPEF, 2004. Disponível em: <http://www.ipef. br/identificacao/cief/>. Acesso em: I 2 maio 201 I.

NISHINO, Y.; JANIN, G.; YAMADA, Y.; KITANO, D. Relations between the colorimetric values and densities of sapwood. Journal of Wood Science, Kyoto, v. 46, p. 267-272, 2000.

PALMA, H. A. L.; LEONELlO, E. C.; BALLARIN, A. W. Demarcação da madeira juvenil e adulta de Corymbia citriodora. Cerne, Lavras, v. I6, p. I4I-I48, 2010.

RIBEIRO, P. G. Utilização de técnicas não destrutivas para caracterização de madeiras de Pinus caribaea var. hondurensis e de Eucalyptus grandis. 2009. II4 f. Dissertação (Mestrado em Ciências Florestais) Universidade de Brasília, Brasília, 2009.

RINK, G. Heartwood color and quantity variation in young black walnut progeny test. Wood Fiber and Science, Madison, v. 19, p. 93-100, 1987.

RINK, G.; PHELPS, J. E. Variation in heartwood and sapwood properties among 10-year-old black walnut trees. Wood Fiber and Science, Madison, v. 21, p. 177-182, 1989.

SOTELO MONTES, C.; HERNÁNDEZ, R. E.; BEAULIEU, J.; WEBER, J. C. Genetic variation in wood color and its correlations with tree growth and wood density of Calycophyllum spruceanum at an early age in the Peruvian Amazon. New Forests, West Lafayette, v. 35, p. 57-73, 2008.

VALENÇA, A. C. V.; PAMPLONA, L. M. P.; SOUTO, S. W. Os novos desafios para a indústria moveleira no Brasil. BNDES Setorial, Rio de Janeiro, n. 15, p. 83-96, 2002.

VANCLAY, J. K.; HENSON, M.; PALMER, G. Color variation and correlations in Eucalyptus dunnii sawnwood. Journal of Wood Science, Kyoto, v. 54, p. 43I-435, 2008. 